\title{
Role of the Noradrenergic System in Synaptic Plasticity in the Hippocampus
}

\author{
Ming-Yuan Min', Hsiu-Wen Yang ${ }^{2}$, and Yi-Wen Lin $^{3}$
}

\section{Summary}

The cortical noradrenergic (NAergic) system, which originates from the locus coeruleus (LC) located in the pons, plays an important role in cortical plasticity and many other brain functions. In rats in which the NAergic system has been eliminated by 6-hydroxydopamine during the neonatal period, induction of longterm potentiation (LTP) at CA1 synapses in the hippocampus is impaired, whereas induction of long-term depression is unaffected. Bath application of norepinephrine, a $\beta$-adrenergic receptor agonist, or activators of effector molecules downstream of the $\beta$-adrenergic receptor restores LTP. Similarly, activation of $\beta$-adrenergic receptors enhances associative LTP induced by paired stimuli to two independent synaptic inputs on the same postsynaptic neuron. The time window within which LTP can be induced by paired stimuli is increased by $\beta$-adrenergic receptor activation, but the magnitude of LTP is not affected. The signaling molecules involved in enhancement of the homosynaptic and associative LTP following $\beta$-adrenergic receptor activation are the same and include protein kinase $\mathrm{A}$ and mitogen-activated protein kinases. These experimental results suggest that a simultaneous increase in the activity of LC neurons during induction protocols may have a permissive role in the induction of homosynaptic and associative LTP in the hippocampus.

Key words LTP, LTD, $\beta$-Adrenergic, 6-Hydroxydopamine, Locus coeruleus

\footnotetext{
${ }^{1}$ Department of Life Science \& Institute of Zoology, College of Life Science, National Taiwan University, No. 1, Sec. 4, Roosevelt Road, Taipei 106, Taiwan

${ }^{2}$ Department of Biomedical Science, Chung-Shan Medical University, No. 110, Sec. 1, Chien-Kuo N. Road, Taichung 402, Taiwan

${ }^{3}$ Institute of Biomedical Science, Academia Sinica, No. 128, Sec. 2, Academia Road, Taipei 115, Taiwan
} 


\section{Introduction}

Long-term potentiation (LTP) and long-term depression (LTD) are use-dependent changes in synaptic efficacy that are generally accepted as the cellular substrate for memory storage in the brain $[1,2]$. They have attracted much attention during the past few decades, and the mechanisms involved in their induction and expression have been well characterized [2-4]. Activation of the $N$-methyl-D-aspartate (NMDA) subtype of glutamate receptors is required for induction of both LTP and LTD. The voltage-dependent nature of the NMDA receptor makes it an ideal detector for the total postsynaptic response produced by a population of activated synapses. These active synapses are potentiated when the total postsynaptic response they produce exceeds a critical value, referred to as the modification threshold $(\theta \mathrm{m})$, and are depressed if the total response is greater than zero but less than $\theta \mathrm{m}[5,6]$.

This idea of regulation of synaptic strength is called the BCM theory, named after the three scientists, Bienenstock, Cooper, and Murp, who proposed it [5]. An example of BCM modification for synaptic efficacy (or BCM curve) at the CA1 synapse is shown in Fig. 1. According to this theory, a total postsynaptic response greater than $\theta \mathrm{m}$ activates large numbers of NMDA receptors, which leads to LTP by the activation of protein kinases, which phosphorylate AMPA receptors. In contrast, a total postsynaptic response greater than zero but less than $\theta \mathrm{m}$ activates fewer NMDA receptors, which leads to LTD because of the activation of phosphatases and the subsequent dephosphorylation of AMPA receptors [4, 7].

Norepinephrine (NE), one of the most important neuronal modulators in the brain, is involved in the regulation of many brain functions, including the wake/

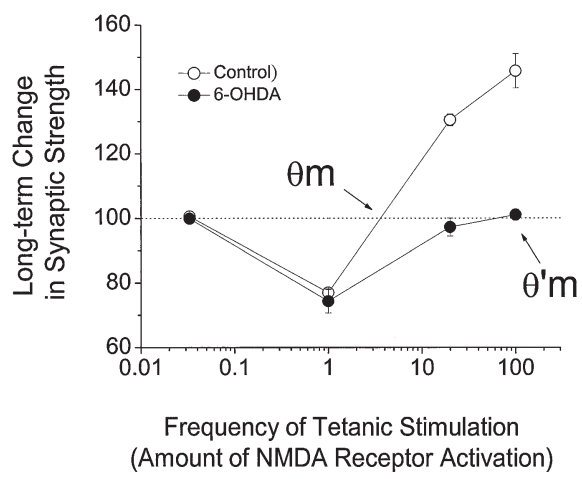

Fig. 1. Effect of norepinephrine (NE) on the BCM curve. The BCM curve shows the relation between the frequency of tetanic stimulation [or the amount of $N$-methyl-D-aspartate (NMDA) receptor activation by tetanic stimulation] and the resultant long-term change in synaptic strength in control slices (open circles). The arrow indicates the modification threshold $(\theta m)$. Note the right shift of $\theta \mathrm{m}$ in the control slices to $\theta^{\prime} \mathrm{m}$ in the 6-hydroxydopamine (6-OHDA)-treated slices (filled circles), suggesting that NE depletion impairs long-term potentiation (LTP) but has no effect on long-term depression (LTD) induction. (From Yang et al. [12], with permission) 
sleep cycle [8], memory storage [9], synaptic/cortical plasticity [10-12], autonomic functions [13], and pain modulation [14]. In the neocortex and hippocampus, NE fibers mainly originate from the locus coeruleus (LC), located in the pons. In vitro studies have suggested that $\mathrm{NE}$, acting on $\beta$-adrenergic receptors, has a significant effect on synaptic plasticity at CA1 synapses $[10,12,15,16]$ and mossy fiber synapses [17]. In this chapter, we first discuss the permissive role of NE in modulating synaptic plasticity based on previous studies, followed by some interesting issues that need to be examined further.

\section{Role of the Noradrenergic System in Homosynaptic Plasticity}

At hippocampal CA1 synapses, bath application of $10 \mu \mathrm{M}$ NE during the delivery of conditioned stimulation to induce a long-term change in synaptic strength results in a left shift of $\theta \mathrm{m}$ in the BCM curve. That is, application of NE does not have any effect on the magnitude of LTP induced by high-frequency stimulation at 50 or $100 \mathrm{~Hz}$ but blocks induction of LTD by low-frequency stimulation $(1 \mathrm{~Hz})$ and enhances the effect of $10-\mathrm{Hz}$ stimulation-which alone does not result in any significant change in synaptic strength in control conditions - to induce LTP [15]. Similar observations have been made at mossy fiber synapses on CA3 pyramidal neurons $[17,18]$. An in vivo study also showed that induction of LTP by mild tetanus stimulation is impaired in animals in which endogenous catecholamine was depleted by injecting 6-hydroxyl dopamine (6-OHDA), but LTP can still be induced by strong tetanus [19]. These observations therefore suggest that the cortical noradrenergic (NAergic) system might have a permissive role in modulating LTP induction in the hippocampus.

Nevertheless, a critical question remains: How do conditioned stimuli for LTP induction activate the NAergic pathway to enhance LTP induction? A high density of NAergic fibers is found in the stratum radiatum of the CA1 region (Fig. 2) and the stratum lucidum of the CA3 region [20, 21]. The overlapping distribution of NAergic and glutamatergic fibers of the Shaffer collateral branches provides an opportunity for simultaneous activation of glutamatergic and NAergic fibers during tetanus. Consistent with this argument, studies in which electrophysiological and neurochemical data were simultaneously recorded in the hippocampus in vivo showed that the local NE concentration can rise to several times the basal value during tetanus for LTP induction [22, 23]. Another potential approach to addressing this question is to examine synaptic plasticity in hippocampal slices from animals in which endogenous NAergic fibers in the hippocampus have been depleted. The treatment of neonatal rats with 6-OHDA might provide a model meeting this requirement, as it results in a persistent loss of catecholaminergic fibers in the cerebral cortex but not in subcortical areas (Fig. 2) [24]. Using hippocampal slices from 6-OHDA-treated rats, we found that the LTP induced by theta burst stimulation decays within $15 \mathrm{~min}$ (Fig. 3A,C), whereas the LTD induced by 900 pulses at $1 \mathrm{~Hz}$ is not affected (Fig. 3B,C) [12]. Taking these results together, it reveals a right 

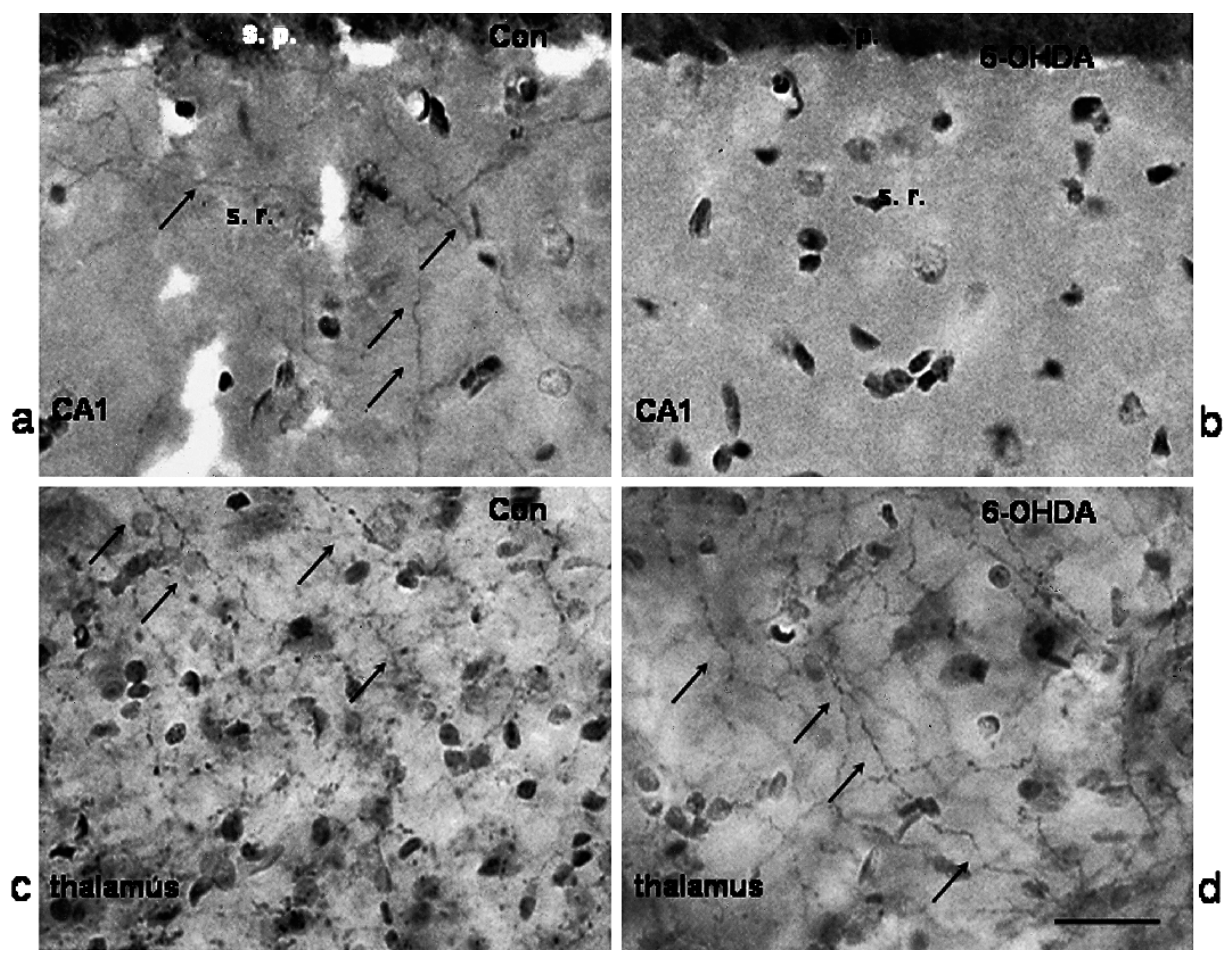

Fig. 2. Animal model in which endogenous NE is depleted by 6-OHDA. Dopamine- $\beta$ hydroxylase $(\mathrm{DBH})$ immunohistochemistry performed in the stratum radiatum (s.r.) of the hippocampal CA1 area in control (Con) (a) and in 6-OHDA-treated animals (b). Thalamus area in the same section in the control animal (c) and the 6-OHDA-treated animal (d). Note the DBHimmunoreactive fibers (indicated by arrows) in all photographs except b. s.p., stratum pyramidale. Bars $50 \mu \mathrm{m}$. (From Yang et al. [12], with permission)

shift of $\theta \mathrm{m}$ in the BCM curve that is consistent with the effect of perfusion slices with $10 \mu \mathrm{M} \mathrm{NE}$ [15]. Interestingly, a similar observation has been reported at mossy fiber synapses, where the LTP induced by mild tetanus decays within $15 \mathrm{~min}$ when $\beta$-adrenergic receptors are blocked by timolol [17]. Bath application of NE restores the expression of LTP to normal and blocks LTD induction (Fig. 3A,B) in slices from 6-OHDA-treated animals but has no effect on the magnitude of LTP in slices from control animals (Fig. 3C) [12]. These observations suggest that NAergic fibers are recruited to enhance LTP induction in normal hippocampal slices, whereas a higher tetanus intensity is required for LTP induction in slices lacking NAergic innervation. Similar conclusions were drawn in a recent study that examined the expression of LTP in several strains of mice with various levels of endogenous NE [16].

The enhancement of LTP and blockage of LTD by NE in the hippocampus appears to occur through the activation of $\beta$-adrenergic receptors [12, 15-17]. In 


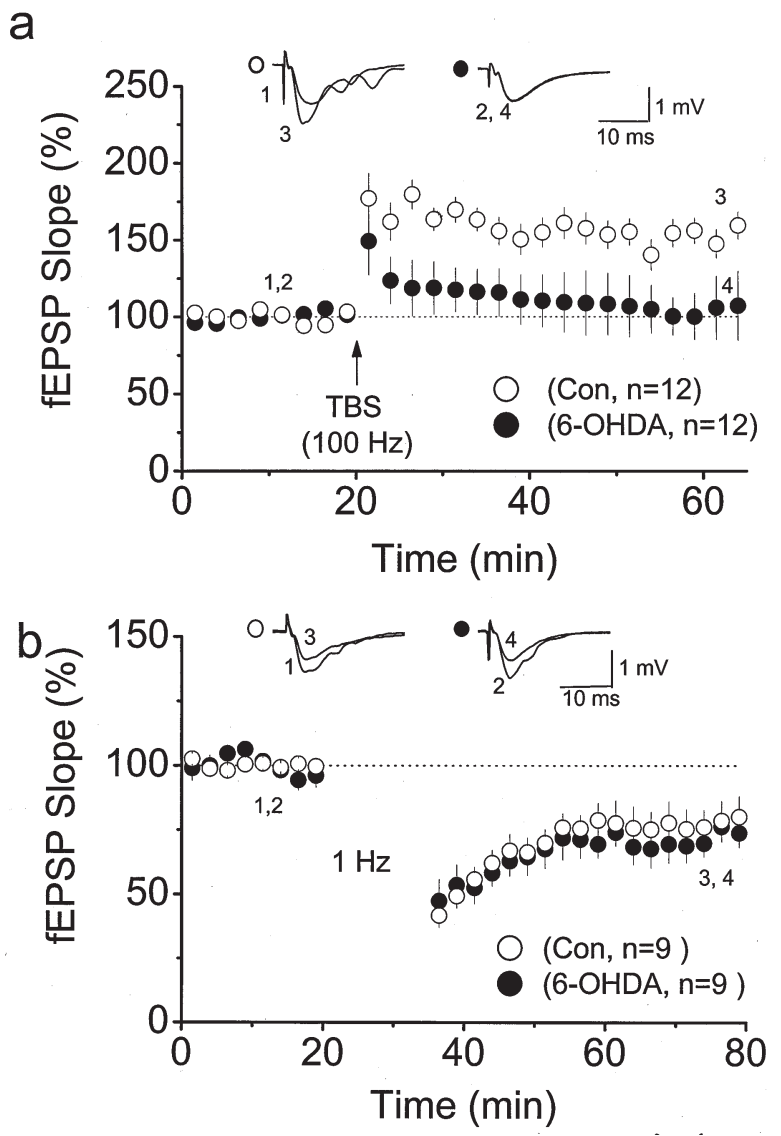

Fig. 3. LTP and LTD in slices from 6-OHDA-lesioned animals. a LTP at CA1 synapses induced in slices of control (open circles) and 6-OHDA-treated (filled circles) rats using theta burst stimulation $(T B S)$, which consists of 10 bursts at $5 \mathrm{~Hz}$ with each burst consisting of four pulses at $100 \mathrm{~Hz}$. b LTD induced by 900 pulses at $1 \mathrm{~Hz}$ stimulation. Note the rapid decay in the LTP, but not the LTD, induced in slices from 6-OHDA-treated rats. (From Yang et al. [12], with permission)

control slices, the effect of NE on the BCM curve is blocked by timolol, a selective $\beta$-adrenergic receptor antagonist, but not by phentolamine, a selective $\alpha$-adrenergic receptor [15]. Similarly, isoproterenol, a selective $\beta$-adrenergic receptor agonist, but not phenylephrine, a selective $\alpha$-adrenergic receptor agonist, restores LTP to normal in slices from 6-OHDA-treated animals (Fig. 4) [12] and from mice with low endogenous NE levels [16]. Interestingly, the blockage of depotentiation (low frequency-induced LTD after LTP induction) at CA1 synapses requires the activation of both $\alpha$ - and $\beta$-adrenergic receptors [15], suggesting that the mechanisms underlying LTD induction and depotentiation are different [25]. 

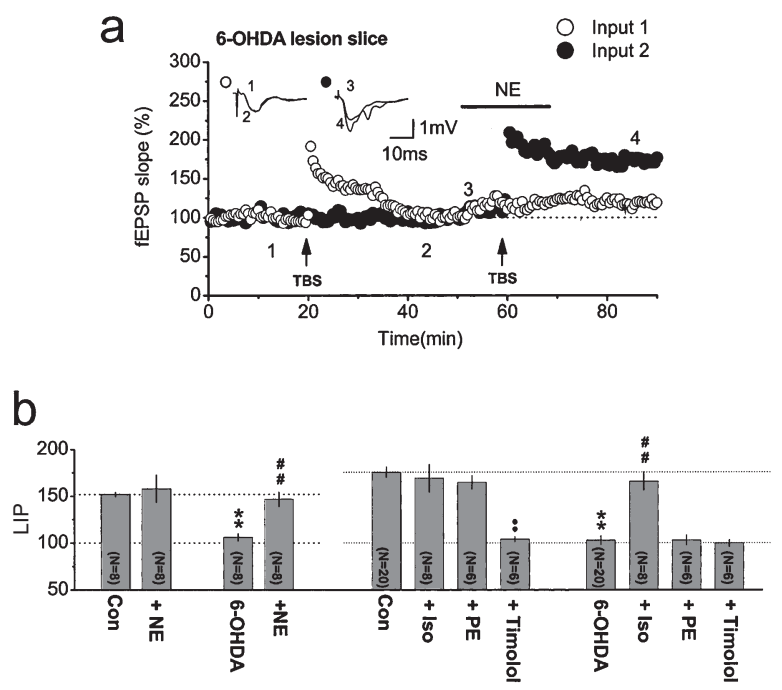

Fig. 4. Activation of $\beta$-adrenergic, but not $\alpha$-adrenergic, receptors restores LTP in slices from 6-OHDA-treated rats. a A typical experiment on a slice from a 6-OHDA-treated rat in which two independent Schaffer collateral branches were stimulated. Theta burst stimulation (TBS) was applied to one of the two inputs (input 1) to induce LTP in normal conditions; $40 \mathrm{~min}$ later, TBS was applied to the other input (input 2) to induce LTP with simultaneous application of NE during delivery of TBS. The resultant LTPs are compared. b Summarized results show that application of NE restores LTP in slices from 6-OHDA-treated rats and the summarized results of pharmacological experiments. Note that in slices from control rats LTP induction is not affected by isoproterenol (Iso), a $\beta$-adrenergic receptor agonist, or phenylephrine (PE), an $\alpha$-adrenergic receptor agonist; it is blocked by timolol, a $\beta$-adrenergic receptor antagonist. Also note in slices from 6-OHDA-treated rats LTP is restored by application of Iso but not PE, and this effect is prevented when the $\beta$-adrenergic receptor is blocked by timolol. (From Yang et al. [12], with permission)

\section{Role of the NAergic System in Associative Plasticity}

A significant weakness in suggesting a physiological role of the conventional LTP and LTD induced by tetanus or prolonged low-frequency stimulation has been the question of whether these stimulating paradigms realistically resemble any physiological function in the brain. Recently, it was demonstrated that LTP/LTDlike changes in synaptic strength can also be induced by the simultaneous spiking of a pair of pre- and postsynaptic neurons within a precise time window, so-called spike timing-dependent plasticity (STDP) [26-28]. Obviously, this form of synaptic plasticity fits very well with the Hebbian learning rules that: (1) neurons that fire in synchrony become wired together (i.e., when the presynaptic axon is active and the postsynaptic neuron simultaneously is strongly activated, the synapse formed by the presynaptic axon is strengthened); and (2) neurons that fire out of synchrony lose their link (see also Chapter 25 in [29]). It is now generally accepted that STDP provides a more genuine cellular model for experience-driven change in brain function or the Hebbian learning rules than does conventional LTP/LTD [26-28, 30, 31]. 
STDP is bidirectional; that is, synaptic efficacy can be either potentiated or depressed by paired pre- and postsynaptic spiking, depending on both the timing interval and the temporal order of the pre- and postsynaptic spiking. Generally speaking, to induce STDP, the timing interval between paired pre- and postsynaptic spiking has to be less than $\sim 25 \mathrm{~ms}$, whereas a significantly wider time window (up to $\sim 100 \mathrm{~ms}$ ) has been suggested for LTD induction [32,33]. As for the temporal order of pre- and postsynaptic spiking, repeated paired pre/postsynaptic spiking results in LTP if presynaptic stimulation precedes postsynaptic stimulation, whereas it results in LTD if the temporal order of pre/postsynaptic spiking is reversed (Fig. 5).

a
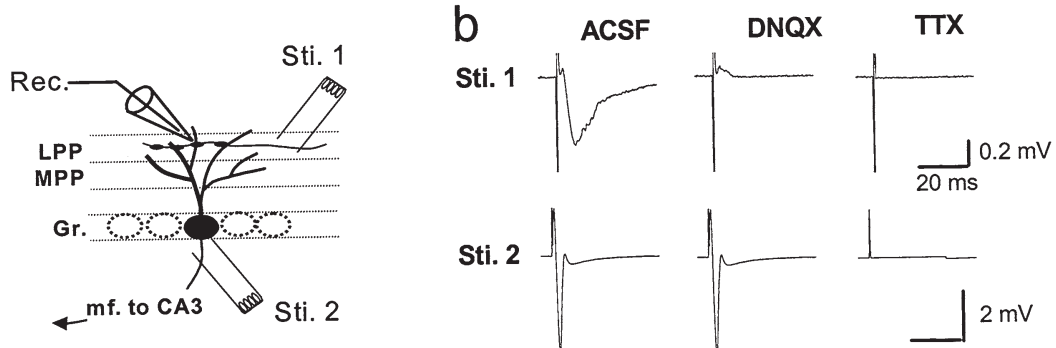

C
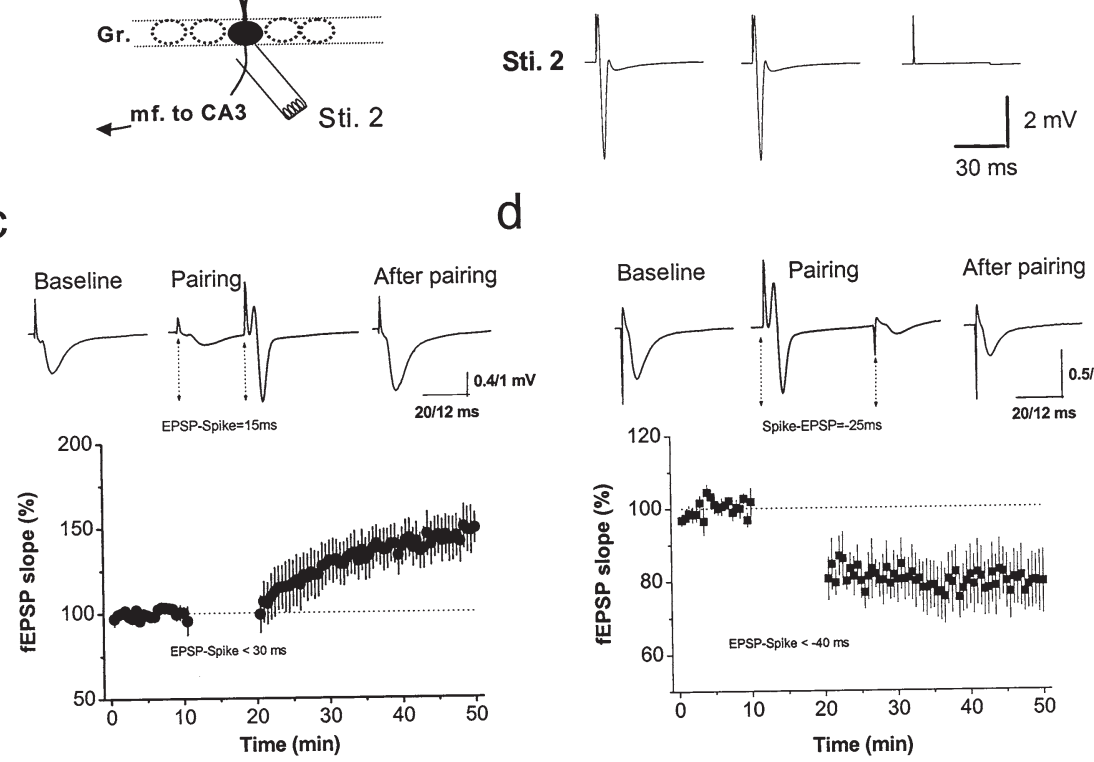

d

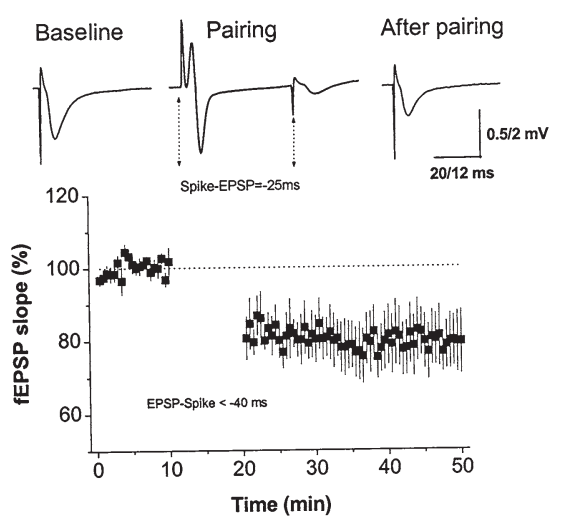

Fig. 5. Spike-timing-dependent plasticity (STDP) at a synapse of the lateral perforant path on a granule cell in the dentate gyrus. a The arrangement of the recording electrode (Rec.) and stimulating electrodes (Sti. 1, Sti. 2). b Evoked neuronal activity by Sti. 1 is field EPSP ( $f E P S P$ ) activity, as it is completed blocked by the AMPA receptor antagonist DNQX. The activity evoked by Sti. 2 is the field somatic spike (fSS), as it is insensitive to DNQX, but is completely blocked by tetrodotoxin (TTX). c LTP induction by paired fEPSP-fSS stimulation. The upper traces show baseline fEPSP activity (left), fEPSP and fSS during pairing (middle), and fEPSP activity after pairing (right) for one experiment. Note the potentiation of fEPSP activity after paired fEPSP-afSS stimulation with $\Delta t=15 \mathrm{~ms}$. The lower plot shows the summarized results for nine experiments, in which the $\Delta t$ for the paired fEPSP-afSS stimulation was $<30 \mathrm{~ms}$. d LTD induction by paired fSS-fEPSP stimulation. Note the depression of fEPSP activity (compare the left and right insets) after paired afSS-fEPSP stimulation with $\Delta t=-25 \mathrm{~ms}$ (see middle inset). The lower plot shows the summarized results for eight experiments, in which $\Delta t$ for the paired fEPSP-afSS stimulation was $<|-40|$ ms. (From Lin et al. [25], with permission) 
a

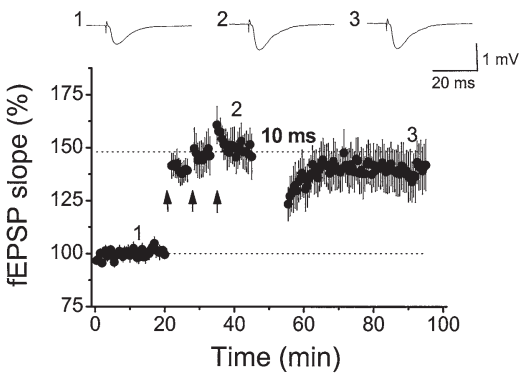

b

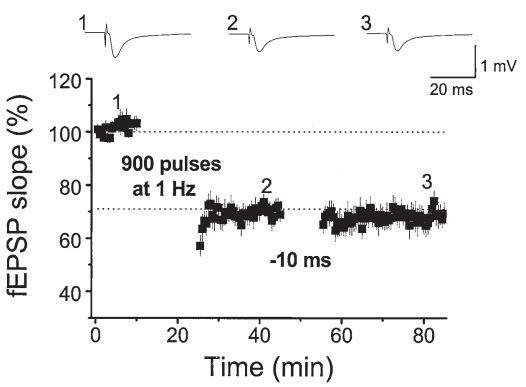

Fig. 6. Induction of STDP is occluded by saturated homosynaptic LTP/LTD. a Homosynaptic LTP induced by three trains of 100 pulses at $100 \mathrm{~Hz}$ (intertrain interval $30 \mathrm{~s}$ ), repeated three times at intervals of $5 \mathrm{~min}$ (arrows); following this homosynaptic LTP, induction of LTP by paired stimulation with an interval of $10 \mathrm{~ms}$ is occluded. b LTD induced by stimulation with 900 pulses at $1 \mathrm{~Hz}$; following this homosynaptic LTD, induction of LTD by paired stimulation with an interval of $-10 \mathrm{~ms}$ is again occluded. (From Lin et al. [25], with permission)

STDP-like LTP and LTD both require activation of NMDA receptors [10, 32, 33]. Again, the voltage-dependent nature of the NMDA receptor makes it an ideal detector for the correlated pre- and postsynaptic spiking activity. Depolarization of the membrane potential by the action potential back-propagated along dendrites of postsynaptic neurons removes magnesium, which blocks the pore site of the NMDA receptor in the resting condition and allows the NMDA receptor to be activated by glutamate released presynaptically during the paired pre- and postsynaptic spiking. We have recently characterized the cellular mechanisms underlying induction of STDP at a synapse of the lateral perforant pathway on a granule cell in the dentate gyrus (Fig. 5). We found that the signaling molecules involved in STDP induction are similar to those involved in conventional LTP and LTD because the saturated synaptic potentiation or depression caused by, respectively, tetanus or low-frequency stimulation, occludes the induction of STDP (Fig. 6) [25]. Similar to the homosynaptic plasticity discussed above, STDP is also subject to modification by the NAergic system. We have reported that by acting at $\beta$-adrenergic receptors NE enhances STDP by increasing the time window of the pre/postsynaptic activation required for LTP induction without changing the magnitude of LTP (Fig. 7) [10]. 

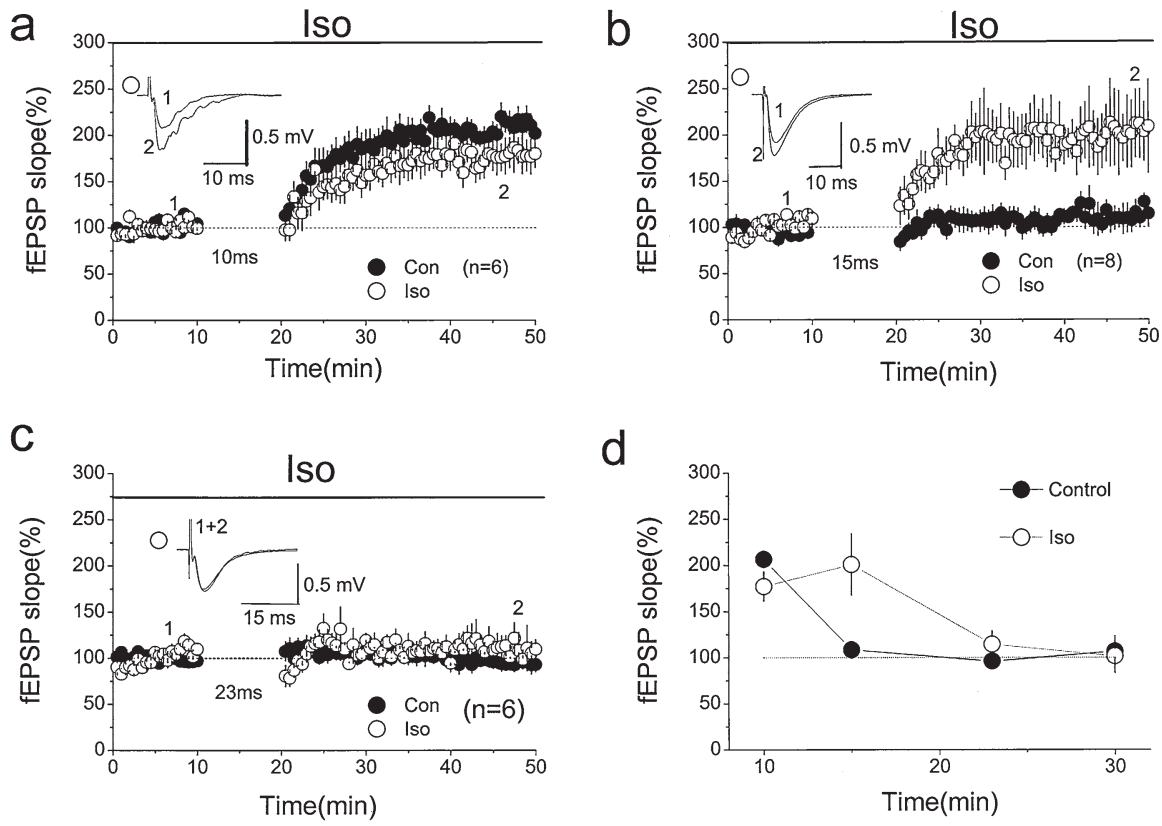

Fig. 7. Enhancement of STDP by activation of $\beta$-adrenergic receptors. a Application of $1 \mu \mathrm{M}$ isoproterenol (Iso) has no significant effect on the magnitude of LTP induced using the pairing protocol with a 10-ms interval. b Application of Iso enhances LTP induction using the pairing protocol with a 15-ms interval, which does not induce LTP under control conditions. c Application of Iso has no significant effect when the interval is increased to $23 \mathrm{~ms}$. d Summarized results for a-c and those using a 30-ms interval. In all of the plots, the open circles are results in the presence of Iso and the filled circles results from control experiments (Con). Note that there is no significant difference in the LTP induced in control and Iso experiments, except for that using a 15-ms interval. (From Lin et al. [10], with permission)

\section{Molecular Signaling Cascades Activated to Enhance LTP}

Activation of $\beta$-adrenergic receptors is known to increase the cytoplasmic concentration of cyclic adenosine monophosphate (cAMP), which in turn activates cAMPdependent protein kinase A (PKA). This signaling pathway seems to act in parallel with the signaling pathway involved in LTP induction, which requires activation of calcium/calmodulin-dependent protein kinase II (CaMKII) by calcium influx through activated NMDA receptors during tetanus [4]. However, several studies have suggested that an increase in cAMP levels in postsynaptic neurons is required for LTP induction under control conditions [34-36]. Consistent with these observations, we found that bath application of activators of Gs protein or adenylyl cyclase (AC) also restored LTP in slices from 6-OHDA-treated animals (Fig. 8) [12]. The type I and VIII ACs, which are present at high amounts in neurons, are good candidate proteins for linking these two pathways. Unlike other AC subtypes, which 
Fig. 8. Membranepermeating cyclic adenosine monophosphate (cAMP) and protein kinase A (PKA) activators restore LTP in slices from 6-OHDA-treated rats. a LTP induction is restored by application of the membrane-permeating cAMP activator, forskolin, at Input 2. b Similar effect of the PKA activators 8-bromocAMP $(n=4)$ and dibutyrylcAMP $(n=4)$ is also observed. The results of these two PKA activators are pooled. (From Yang et al. [12], with permission)
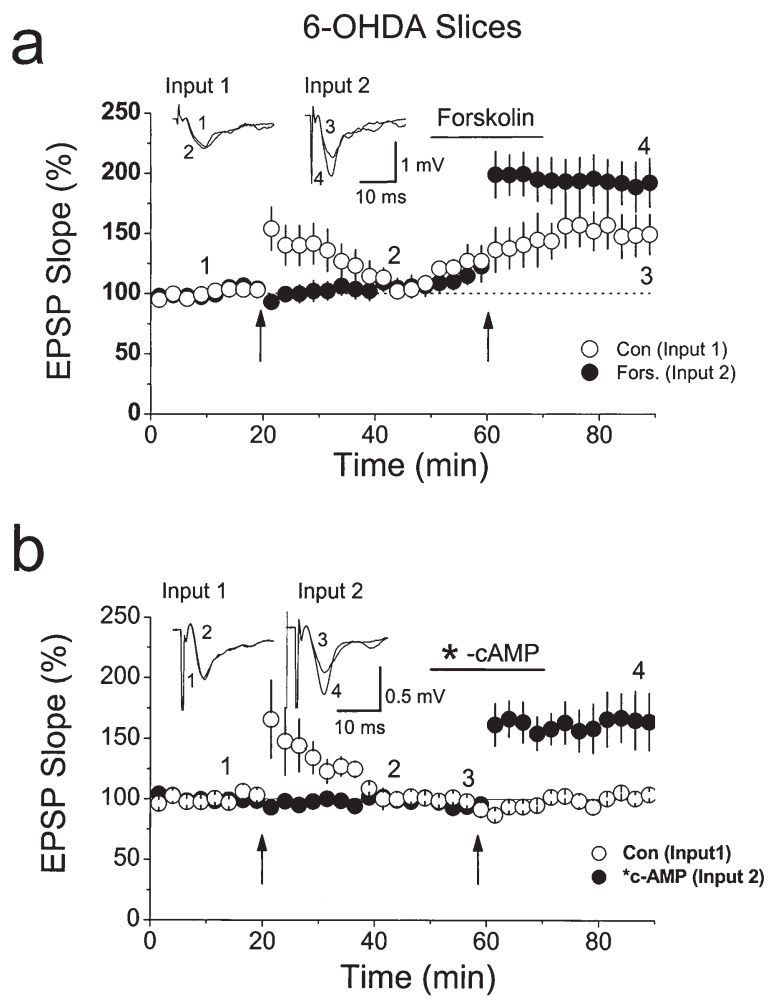

require an external signal for their activation, types I and VIII can be directly activated by CaMKII intracellularly and contribute to the cAMP increase in the cytoplasm [37]. Thus, the cAMP increase required for LTP induction might occur via activation of type I or VIII ACs by CaMKII $[38,39]$. In hippocampal slices taken from type VIII AC knockout mice, induction of LTP is impaired, demonstrating the involvement of this protein in LTP induction [40].

It is likely that the permissive effect of the $\beta$-adrenergic receptor on homosynaptic LTP induction might be through the activation of other types of ACs downstream of the Gs proteins activated by $\beta$-adrenergic receptors, which in turn cause a sufficiently large increase in cytoplasmic cAMP for LTP induction. Therefore, cooperative activation of other types ACs by $\beta$-adrenergic receptors with activation of types I and VIII by NMDA receptor signaling leads to LTP induction. This could also explain why, in hippocampal slices that lack NAergic fiber innervation, strong tetanus is required for LTP induction, as more type I or VIII ACs must be activated to produce enough cAMP for LTP induction.

At hippocampal CA1 synapses, the expression of LTP is due to enhancement of AMPA receptor function, either by phosphorylation of existing AMPA receptors at synaptic sites or by recruitment of new AMPA receptors to the synaptic activation zone [41]. In addition to being directly involved in modulation of AMPA 


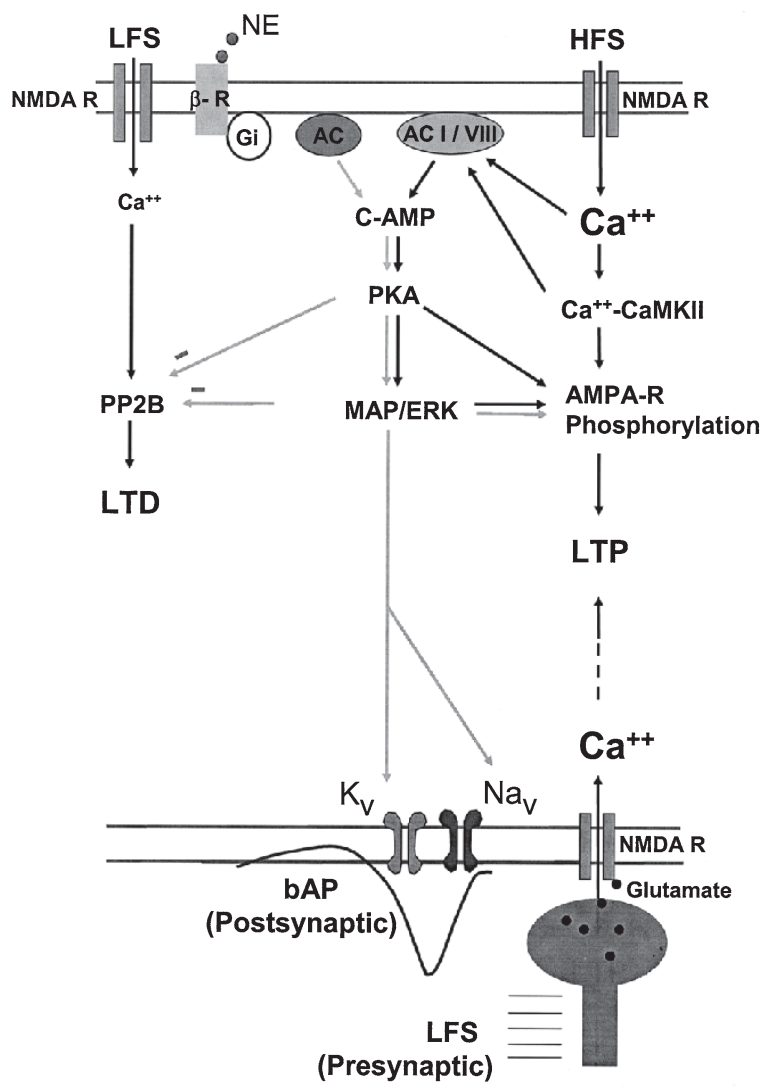

Fig. 9. Signaling cascades involved in the enhancing effect of $\beta$-adrenergic receptors $(\beta-R)$ on synaptic plasticity at CA1 synapses. Top right High-frequency stimulation (HFS) activates many NMDA receptors (NMDA $R$ ) and causes a large amount of calcium influx and activation of CaMKII. The activated CaMKII either phosphorylates the AMPA receptor (AMPA-R) itself, leading to LTP, or increases cytoplasmic levels of cAMP by stimulating type I or VIII ACs (ACI/VIII), which activate PKA or the more downstream effectors MAP/ERK to cause phosphorylation of AMPA receptors and LTP induction (black arrows). Middle Activation of $\beta$-adrenergic receptors increases cytoplasmic levels of cAMP to enhance LTP induction, either by stimulating type I/VIII ACs or other types of AC (gray arrows). Activation of $\beta$-adrenergic receptors also inhibits phosphatase 2B (PP2B) and prevents induction of LTD by low-frequency stimulation $(L F S)$ (bottom). As regards STDP, paired pre-/postsynaptic spiking causes activation of many NMDA receptors and leads to LTP as homosynaptic LTP. In addition, the activated PKA-MAP/ ERK signaling pathway also modulates voltage-dependent potassium $\left(K_{v}\right)$ and sodium $\left(N a_{v}\right)$ channels function to shape the back-propagating action potential $(b A P)$, which in turn changes the time window for LTP induction

receptor function, the signaling molecules activated following $\beta$-adrenergic receptor activation must also target other ion channels or receptors to enhance the associative form of LTP or STDP. This is because $\beta$-adrenergic receptor activation does not affect the magnitude of LTP but increases the time window of the pre/postsynaptic activation required for LTP induction (Fig. 9) [10]. For example, one possible candidate molecule is the $\mathrm{Kv} 4.3$ channel, which is responsible for a transient (or 
A-type) potassium current reported to play an important role in controlling backpropagation of the action potential along dendrites in pyramidal neurons in the hippocampus [42]. Kv4.3 channel function is also subject to regulation by activation of $\beta$-adrenergic receptors through the phosphorylation of the Kv4.3 channels by PKA [42]. As a result, the time window of the pre/postsynaptic activation required for LTP induction is changed because of the changed properties of the back-propagated postsynaptic spike. Another possibility is that $\beta$-adrenergic receptors are located on the presynaptic terminals, and their activation results in a change in the profile of glutamate release presynaptically, which in turn changes the time window of pre/postsynaptic activation.

According to the BCM theory, during prolonged low-frequency stimulation fewer NMDA receptors are activated, there is less calcium influx into the postsynaptic cytoplasm, and phosphatase $2 \mathrm{~B}$ (or calcineurin) is activated, rather than CaMKII, causing dephosphorylation of existing AMPA receptors and resulting in LTD [4]. Activation of the $\beta$-adrenergic receptor-cAMP-PKA pathway activates inhibitor I, which inhibits calcineurin (phosphatase 2B) and blocks LTD [4, 43, 44]. Interestingly, LTD induction is not affected in slices from 6OHDA-treated animals [12], suggesting that NAergic fibers are not recruited during low-frequency stimulation, which may be due to their higher threshold for activation. Consistent with this argument, we found that STDP induction is also not affected [10], as presynaptic stimulation is given at low frequency during its induction.

\section{Other Considerations}

If recruitment of NAergic fibers occurs only during tetanus stimulation, which does not resemble any physiological condition in the neuronal circuit, how is the NAergic system activated to modulate induction of homosynaptic plasticity and STDP under physiological conditions? Neuronal activity of LC neurons undergoes certain rhythms, which are closely related to the wake/sleep cycle of animals. They fire at highest frequency when animals are active waking, less when animals are quiet waking, and even less when animals are in non-rapid eye movement (REM) sleep and REM sleep [45, 46]. Thus, synaptic plasticity would be enhanced if an increase in synaptic activity in hippocampal circuit occurred simultaneously with an increase in the spiking rate of LC neurons; for example, synaptic plasticity might occur more easily during active waking. Sleep, however, has been suggested to play a significant role in declarative memory consolidation [47], as sleep deprivation has been shown to impair long-term synaptic plasticity significantly in the hippocampus in rats $[48,49]$. These observations suggest that the temporal dissociation of NAergic activity from the neuronal circuit in the cortex and hippocampus during sleep could be essential for memory consolidation.

Like memory, LTP expression has different stages. There is an early-phase LTP (e-LTP), which usually lasts only 3-4h after its induction and is independent 
of protein synthesis. Following e-LTP is late-phase LTP (1-LTP), which can last several hours in slice preparations or even days in vivo and is dependent on protein synthesis $[50,51]$. At the cellular level, e-LTP might resemble the acquisition of new memory, and the conversion of e-LTP to l-LTP might resemble memory consolidation. As discussed above, the NAergic system, acting on $\beta$-adrenergic receptors, may have a permissive effect on the induction of e-LTP, and it may have a similar effect on memory acquisition. In line with this argument, a behavioral study showed that coeruleocortical NAergic lesions produced by intracerebral injection of 6-OHDA impairs learning behavior in animals [52]. What about the effect of the NAergic system on 1-LTP? Does it also enhance 1-LTP expression? Given that sleep is important for memory consolidation, which resembles the conversion of e-LTP to 1-LTP at the cellular level, and that neuronal activity of LC neurons is low during sleep, it might appear that lower endogenous levels of NE would be the ideal situation for e-LTP to convert to l-LTP. However, experimental results do not support this argument. At mossy fiber synapses, homosynaptic e-LTP and 1-LTP are both enhanced by $\beta$-adrenergic receptor activation [17]. One important factor that should be borne in mind is that mossy fiber LTP is NMDA receptor-independent [53, 54]; furthermore, the effect of endogenous levels of NE must be taken into account. Thus, at CA1 synapses, where LTP is NMDA receptor-dependent, the role of the NAergic system on 1-LTP requires further investigation.

It has been shown that slow-wave sleep and sleep spindles are possible candidate mechanisms for sleep to enhance memory directly [55]. In hippocampal slices, it is also been demonstrated that somatic spiking at theta frequency [56] or ripple complex activity can directly enhance the conversion of e-LTP to 1-LTP [57]. Thus, at the cellular and molecular levels, changes in the synchronization of the neuronal spiking pattern might be a crucial factor in enhancing expression of 1-LTP and memory. Thalamic neurons can change their spiking patterns and degree of synchronized firing depending on the extracellular levels of NE, and the modification of the functions of ionic channels by NE can account for the changed spiking activity [52]. If the NAergic system did have a significant effect on 1-LTP, would it involve a similar mechanism and allow somatic activity-dependent modification of 1-LTP? Again, this would be an interesting question to answer.

\section{Conclusions}

A significant role of the NAergic system in modulating homosynaptic and associative forms of synaptic plasticity has been confirmed and the underlying molecular mechanism explored in detail. However, some questions remain to be answered, in particular the possible differential effects of the NAergic system on cortical plasticity during different states of brain functions, for example, during active awaking and/or sleep. 


\section{References}

1. Bliss TVP, Collingridge GL (1993) Synaptic model of memory: long term potentiation in the hippocampus. Nature 361:31-39.

2. Malenka RC, Nicoll RA (1999) Long term potentiation: a decade of progress? Science 285:1870-1875.

3. Bear MF, Abraham WC (1996) Long-term depression in hippocampus. Annu Rev Neurosci 19:437-462.

4. Roberson ED, English JD, Sweatt JD (1996) Second messengers in LTP and LTD. In: Fazeli MS, Collingridge GL (eds) Cortical plasticity: LTP and LTD. BIOS Scientific, Oxford, pp 35-60.

5. Bienenstock L, Cooper NL, Munro PW (1982) Theory for the development of neuron selectivity: orientation specificity and binocular interaction in visual cortex. J Neurosci 2:32-48.

6. Bear MF, Kirkwood A (1996) Bidirectional plasticity of cortical synapses. In: Fazeli MS, Collingridge GL (eds) Cortical plasticity: LTP and LTD. BIOS Scientific, Oxford, pp 191-205.

7. Bear MF, Malenka RC (1994) Synaptic plasticity: LTP and LTD. Curr Opin Neurol 4:389-399.

8. Aston-Jones G, Cohen JD (2005) An integrative theory of locus coeruleus-norepinephrine function: adaptive gain and optimal performance. Annu Rev Neurosci 28:403-450.

9. Selden NR, Robbins TW, Everitt BJ (1990) Enhanced behavioral conditioning to context and impaired behavioral and neuroendocrine response to conditioned stimuli following ceruleocortical noradrenergic lesion: support for an attentional hypothesis of central noradrenergic function. J Neurosci 10:531-539.

10. Lin Y-W, Min M-Y, Chiu T-H, et al (2003) Enhancement of associative long-term potentiation by activation of $\beta$-adrenergic receptors at CA1 synapses in rat hippocampal slices. $\mathrm{J}$ Neurosci 23:4173-4181.

11. Kasamatsu T (1991) Adrenergic regulation of visual cortical plasticity; a role of locus coeruleus system. Prog Brain Res 88:599-616.

12. Yang H-W, Lin Y-W, Yen C-D, et al (2002) Change in bi-directional plasticity at CA1 synapses in hippocampal slices taken from 6-hydroxy-dopamine treated rats: the role of endogenous norepinephrine. Eur J Neurosci 16:1117-1128.

13. Richerson GB (2003) The autonomic nervous system. In: Boron WF, Boulpaep EL (eds) Medical physiology. Saunders, Philadelphia, pp 378-398.

14. Pertovaara A (2006) Noradrenergic pain modulation. Prog Neurobiol 80:53-83.

15. Katsuki H, Izumi Y, Zorumski CF (1997) Noradrenergic regulation of synaptic plasticity in the hippocampal CA1 region. J Neurophysiol 77:3013-3020.

16. Schimanski LA, Ali DW, Baker GB, et al (2007) Impaired hippocampal LTP in inbred mouse strains can be rescued by $\beta$-adrenergic receptor activation. Eur J Neurosci 25:1589-1598.

17. Huang Y-Y, Kandel ER (1996) Modulation of both the early and the late phase of mossy fiber LTP by activation of $\beta$-adrenergic receptors. Neuron 16:611-617.

18. Hopkins W, Johnston D (1988) Noradrenergic enhancement of long-term potentiation of mossy fiber synapses in the hippocampus. J Neurophysiol 59:667-678.

19. Bliss TVP, Goddard GV, Riives M (1983) Reduction of long-term potentiation in the dentate gyrus of the rat following selective depletion of monoamines. J Physiol (Lond) 334: 475-491.

20. Loy R, Koziell DA, Lindsey D, et al (1980) Noradrenergic innervation of adult rat hippocampal formation. J Comp Neurol 189:699-710.

21. Moore RY, Bloom FE (1979) Central catecholamine neuron systems: anatomy and physiology of the norepinephrine and epinephrine systems. Annu Rev Neurosci 2:113-168.

22. Bronzino JD, Kehoe P, Mallinson K, et al (2001) Increased extracellular release of hippocampal NE is associated with tetanization of the medial perforant pathway in the freely moving adult male rat. Hippocampus 11:423-429. 
23. Harley CW, Lalies MD, Nutt DJ (1996) Estimating the synaptic concentration of norepinephrine in dentate gyrus which produces beta receptor mediated long-lasting potentiation in vivo using microdialysis and intracerebroventricular norepinephrine. Brain Res 710:293-298.

24. Harik SI (1984) Locus coeruleus lesion by local 6-hydroxydopamine infusion causes marked and specific destruction of noradrenergic neurons, long term depletion of norepinephrine and enzymes that synthesize it, and enhanced dopaminergic mechanisms in the ipsilateral cerebral cortex. J Neurosci 4:699-707.

25. Lin Y-W, Yang H-W, Wang H-J, et al (2006) Spike-timing-dependent plasticity (STDP) at resting and conditioned lateral perforant path synapses on granule cells in the dentate gyrus: different roles of NMDA and group I metabotropic glutamate receptors. Eur J Neurosci 23:2362-2374.

26. Bi G, Poo M-M (2001) Synaptic modification by correlated activity: Hebb's postulate revised. Annu Rev Neurosci 24:139-166.

27. Dan Y, Poo M-M (2004) Spike-timing-dependent plasticity of neuronal circuits. Neuron 44:23-30.

28. Dan Y, Poo MM (2006) Spike timing-dependent plasticity: from synapse to perception. Physiol Rev 863:1033-1048.

29. Bea MF, Connors BW, Paradiso MA (2007) Neuroscience: exploring the brain (3rd ed). Lippincott Williams \& Wilkins, Philadelphia.

30. Song S, Abbott LF (2001) Cortical development and remapping through spike timingdependent plasticity. Neuron 32:339-350.

31. Song S, Miller KD, Abbott LF (2000) Competitive Hebbian learning through spiketiming-dependent plasticity. Nat Neurosci 3:919-926.

32. Debanne D, Gahwiler BH, Thomson SM (1998) Long term synaptic plasticity between pairs of individual CA3 pyramidal cells in rat hippocampal slice cultures. J Physiol (Lond) 507:237-247.

33. Feldman DE (2000) Timing-based LTP and LTD at vertical inputs to layer II / III pyramidal cells in rat barrel cortex. Neuron 27:45-56.

34. Chetaovich DM, Sweatt JD (1993) NMDA receptor activation increases cyclic AMP in area CA1 of the hippocampus via calcium/calmodulin stimulation of adenylyl cyclase. J Neurochem 61:1933-1942.

35. Makhinson M, Chotiner JK, Watson JB, et al (1999) Adenylyl cyclase activation modulates activity-dependent changes in synaptic strength and $\mathrm{Ca}^{2-} /$ calmodulin-dependent kinase II autophosphorylation. J Neurosci 19:2500-2510.

36. Otmakhova NA, Otmakhov N, Mortenson LH, et al (2000) Inhibition of cAMP pathway decreases early long-term potentiation at CA1 hippocampal synapses. J Neurosci 20:4446-4451.

37. Cooper DMF, Mons N, Karpen JW (1995) Adenylyl cyclase and the interaction between calcium and cAMP signalling. Nature 374:421-424.

38. Liauw J, Wu LJ, Zhuo M (2005) Calcium-stimulated adenylyl cyclases required for long-term potentiation in the anterior cingulate cortex. J Neurophysiol 94:878-882.

39. Wang H, Ferguson GD, Pineda VV, et al (2004) Overexpression of type-1 adenylyl cyclase in mouse forebrain enhances recognition memory and LTP. Nat Neurosci 7: 635-642.

40. Wang H, Pineda VV, Chan GC, et al (2003) Type 8 adenylyl cyclase is targeted to excitatory synapses and required for mossy fiber long-term potentiation. J Neurosci 23:9710-9718.

41. Malenka RC, Nicoll RA (1997) Silent synapses speak up. Neuron 19:473-476.

42. Yuan LL, Adams JP, Swank M, et al (2002) Protein kinase modulation of dendritic $\mathrm{K}^{-}$ channels in hippocampus involves a mitogen-activated protein kinase pathway. J Neurosci 22:4860-4868.

43. Mulkey RM, Endo S, Shenolikar S, et al (1994) Involvement of a calcineurin/inhibitor-1 phosphatase cascade in hippocampal long-term depression. Nature 369:486-488.

44. Mulkey RM, Herron CE, Malenka RC (1993) An essential role for protein phosphatases in hippocampal long term depression. Science 261:1051-1055. 
45. Aston-Jones G, Bloom FE (1981) Activity of norepinephrine-containing locus ceoruleus neuron in behaving rats anticipates fluctuation in sleep-waking cycle. J Neurosci $1: 876-886$.

46. Foote SL, Aston-Jones G, Bloom FE (1980) Impulse activity of locus coeruleus neurons in awake rats and monkeys is a function of sensory stimulation and arousal. Proc Natl Acad Sci U S A 77:3033-3037.

47. Ellenbogen JE, Payne JD, Stickgold R (2006) The role of sleep in declarative memory consolidation: passive, permissive, active or none? Curr Opin Neurobiol 16:716-722.

48. Ishikawa A, Kanayama Y, Matsumura H, et al (2006) Selective rapid eye movement sleep deprivation impairs the maintenance of long-term potentiation in the rat hippocampus. Eur $\mathrm{J}$ Neurosci 24:243-248.

49. Kopp C, Longordo F, Nicholson JR, et al (2006) Insufficient sleep reversibly alters bidirectional synaptic plasticity and NMDA receptor function. J Neurosci 26:12456-12465.

50. Frey U, Huang YY, Kandel ER (1993) Effects of cAMP stimulate a late stage of LTP in hippocampal CA1 neuron. Science 260:1661-1664.

51. Huang YY, Kandel ER (1994) Recruitment of long lasting and protein kinase A dependent long term potentiation in CA1 region of hippocampus requires repeated tetanization. Learn Mem 1:74-82.

52. Steriade M, McCarley RW (1990) Brainstem control of wakefulness and sleep. Plenum, New York.

53. Weisskopf MG, Castillo PE, Zalutsky RA, et al (1994) Mediation of hippocampal mossy fiber long-term potentiation by cyclic AMP. Science 265:1878-1882.

54. Weisskopf MG, Nicoll RA. (1995) Presynaptic changes during mossy fibre LTP revealed by NMDA receptor-mediated synaptic responses. Nature 376:256-259.

55. Gais S, Molle M, Helms K, et al (2002) Learning-dependent increases in sleep spindle density. J Neurosci 22:6830-6834.

56. Dudek SM, Fields RD (2002) Somatic action potential are sufficient for late-phase LTPrelated cell signaling. Proc Natl Acad Sci U S A 99:3962-3967.

57. Behrens CJ, van den Boom LP, de Hoz L, et al (2005) Induction of sharp wave-ripple complexes in vitro and reorganization of hippocampal networks. Nat Neurosci 8:1560-1567. 